\title{
SOME EQUIDISTRIBUTED STATISTICS ON GENOCCHI PERMUTATIONS
}

BY

Arthur RANDRIANARIVONY and Jiang ZENG

Département de Mathématiques, Université Louis Pasteur

7, rue René Descartes, 67084 Strasbourg Cedex, France

Dedicated to Dominique FOATA on the occasion of his sixtieth birthday

Submitted: February 1, 1995; Accepted: January 14, 1996

Abstract. A Genocchi permutation $\sigma$ of $[2 n]$ is a permutation such that $\sigma(2 i-1)>2 i-1, \quad \sigma(2 i) \leq 2 i$ for all $i \in\{1,2, \ldots, 2 n\}$. The purpose of this paper is to investigate the equidistribution of several bivariate statistics on the Genocchi permutations.

\section{Introduction.}

Let $\mathcal{S}_{n}$ be the set of permutations on $\{1,2, \ldots, n\}$. Recall that a permutation $\sigma \in \mathcal{S}_{2 n}$ is a Genocchi permutation if $\sigma(2 i-1)>2 i-1$ and $\sigma(2 i) \leq 2 i$ for all $i \in\{1,2, \ldots, n\}$. The set of the Genocchi permutations on $\{1,2, \ldots, 2 n\}$ will be denoted by $\mathcal{G}_{2 n}$. The concept of Genocchi permutation was introduced by Dumont [D], back in 1974, who proved that $\# \mathcal{G}_{2 n}$, i.e., the number of Genocchi permutations on $\{1,2, \ldots, 2 n\}$, was equal to the Genocchi number $G_{2 n+2}$. Recall that the exponential generating function for the (signed) Genocchi numbers $G_{2 n}$ reads

$$
t+\sum_{n \geq 1}(-1)^{n} G_{2 n} \frac{t^{2 n}}{(2 n) !}=\frac{2 t}{e^{t}+1} .
$$

The first values of those numbers appear in the next table

\begin{tabular}{|c|c|c|c|c|c|c|c|c|c|}
\hline$n$ & 1 & 2 & 3 & 4 & 5 & 6 & 7 & 8 & 9 \\
\hline$G_{2 n}$ & 1 & 1 & 3 & 17 & 155 & 2073 & 38227 & 929569 & 28820619 \\
\hline
\end{tabular}

The Genocchi permutations play for the Genocchi numbers the role played by the alternating permutations for the Euler numbers $E_{n}$. They are indeed also alternating, but their graph, represented in the square $[n] \times[n]$, 
crosses the diagonal of that square a maximum number of times. The numbers $E_{n}$ are the coefficients of the Taylor expansion of the function $\tan t+\sec t$. It was shown by André [A], already in 1881, that $E_{n}$ was equal to the number of alternating permutations on $[n]$. Since then, several studies on the alternating permutations have been made: see, e.g., [F-S, C-S, V, R-Z1] and therefore several refinements of the Euler numbers have been obtained.

A parallel work on the Genocchi permutations is still in its infancy. Much has been done on the surjective pistol set-up (see the definition further on in the paper) to derive original results on several classes of polynomials connected with the Genocchi numbers (see [D, D-R, R-Z2]), but little has been done on the Genocchi permutations proper. However the first author of the paper has recently obtained the continued fraction expansion of the generating function for Genocchi permutations by number of fixed points and inversion number [R2].

The purpose of the paper is to present a new result on Genocchi permutations by defining three pairs of statistics and showing that they are equidistributed. The definitions of our statistics on $\mathcal{G}_{2 n}$ are refinements of classical statistics that have been thoroughly studied on $\mathcal{S}_{2 n}$ (see [F-S]). They are defined as follows.

Let $\sigma=\sigma(1) \sigma(2) \ldots \sigma(n)$ be a permutation on $\{1, \ldots, n\}$. The value $\sigma(i)(1 \leq i \leq n)$ is said to be a fixed point, if $\sigma(i)=i$; a left-to-right maximum, if $\sigma(j)<\sigma(i)$ for all $j<i$; a right-to-left minimum, if $\sigma(i)<$ $\sigma(j)$ for all $j>i$. Denote by fix $\sigma$ (resp. lma $\sigma$, resp. rmi $\sigma$ ) the number of fixed points (resp. of left-to-right maxima, resp. of right-to-left minima) of $\sigma$.

The left-to-right maxima and right-to-left minima are further discriminated according to their parities. We denote by lema $\sigma$ (resp. remi $\sigma$, resp. romi $\sigma$ ) the number of even left-to-right maxima (resp. of even rightto-left minima, resp. of odd right-to-left minima) of $\sigma$. We will not make use of the number of odd left-to-right maxima.

Finally, we say that $\sigma(i)$ is a cycle minimum (resp. cycle maximum) of $\sigma$, if $\sigma(i)$ is the minimum (resp. maximum) in its cycle (i.e., its orbit). Let comi $\sigma$ (resp. cemi $\sigma$ ) be the number of odd (resp. even) cycle minima of $\sigma$ and $\operatorname{cmax} \sigma$ be the number of cycle maxima of $\sigma$ which are not fixed points.

Example The Genocchi permutation

$$
\sigma=\left(\begin{array}{cccccccc}
1 & 2 & 3 & 4 & 5 & 6 & 7 & 8 \\
4 & 2 & 7 & 1 & 6 & 5 & 8 & 3
\end{array}\right) \in \mathcal{G}_{8}
$$

has one fixed point, 2 ; three left-to-right maxima, 4, 7, 8 (only two of them are even); two right-to-left minima, 3, 1 (and they are both odd). The cycle form $(14)(2)(378)(56)$ shows that there are three odd cycle minima, $1,3,5$; one even cycle minimum, 2 and three cycle maxima different from 
cycle minima, 4, 6, 8. Accordingly, fix $\sigma=1$, lema $\sigma=2$, remi $\sigma=0$, $\operatorname{romi} \sigma=2$, rmi $\sigma=2$, comi $\sigma=3$, cemi $\sigma=1$ and $\operatorname{cmax} \sigma=3$.

For each Genocchi permutation $\sigma$ we have fix $\sigma=\operatorname{cemi} \sigma$, since each even cycle-minimum, say $\sigma(j)=2 i$, of $\sigma$ must satisfy $\sigma(2 i) \geq 2 i$, because it is a minimum in its cycle and also $\sigma(2 i) \leq 2 i$ by definition of a Genocchi permutation. The main result of this paper is the following theorem.

Theorem 1. The three bivariate statistics (comi, cemi), (lema, fix) and (remi, romi) are equidistributed on $\mathcal{G}_{2 n}$, i.e,

$$
\sum_{\sigma \in \mathcal{G}_{2 n}} x^{\text {comi } \sigma} y^{\text {cemi } \sigma}=\sum_{\sigma \in \mathcal{G}_{2 n}} x^{\text {lema } \sigma} y^{\text {fix } \sigma}=\sum_{\sigma \in \mathcal{G}_{2 n}} x^{\text {romi } \sigma} y^{\text {remi } \sigma} .
$$

An ideal proof of (1.1) would be a proof based on two weight preserving bijections on $\mathcal{G}_{2 n}$ which transfer the pair (comi, cemi) to (lema, fix) and to (romi, remi) respectively. Our proof will use both analytical and combinatorial methods. In fact, the proof of the first equality of Theorem 1 consists essentially of showing that the generating polynomials of $\mathcal{G}_{2 n}$ with respect to (comi, cemi) and (lema, fix) both satisfy the following recurrence relation :

$$
\left\{\begin{array}{l}
D_{1}(x, y)=x \\
D_{n}(x, y)=x(y+1) D_{n-1}(x+1, y+1)-x y D_{n-1}(x, y) .
\end{array}\right.
$$

We note that the above polynomials are essentially the the same polynomials $B_{n}(x, y)=x^{-1} D_{n}(x, y)(n \geq 1)$ introduced by Dumont and Randrianarivony [D-R1] as an extension of Gandhi's polynomials $D_{n}(x, x)$ [D].

In section 2 , we prove bijectively that the generating polynomial of $\mathcal{G}_{2 n}$ with respect to (comi, cemi) satisfies recurrence (1.2). In section 3 , we expand the ordinary generating function of the enumerating polynomials of $\mathcal{G}_{2 n}$ with respect to four statistics (lema, romi, fix, remi) into a Stieltjes continued fraction. In section 4 , we apply a result established in [R-Z] to show that the generating polynomials of $\mathcal{G}_{2 n}$ with respect to (lema, fix) and (romi, remi) both satisfy the recurrence (1.2). Finally, a conjectured extension of Theorem 1 is also formulated.

\section{Combinatorial interpretations of $D_{n}(x, y)$.}

Recall that a surjective pistol $p$ on $\{1,2, \ldots, 2 n\}$ is a surjective mapping $p:\{1,2, \ldots, 2 n\} \rightarrow\{2,4, \ldots, 2 n\}$ such that $p(2 i), p(2 i-1) \geq 2 i$ for all $i \in\{1,2, \ldots, n\}$. Let $\mathcal{P}_{2 n}$ be the set of surjective pistols on $\{1,2, \ldots, 2 n\}$. For $p \in \mathcal{P}_{2 n}$, we denote by maxi $p($ resp. $\operatorname{maxp} p)$ the number of odd (resp. even) integers $k \in\{1,2, \ldots, 2 n-1\}$ such that $p(k)=2 n$. 
Proposition 2. For $n \geq 1$ we have

$$
D_{n}(x, y)=\sum_{p \in \mathcal{P}_{2 n}} x^{\operatorname{maxi} p} y^{\operatorname{maxp} p}
$$

Proof: This can be easily verified by induction on $n$ as Dumont [D] did for Gandhi polynomials $D_{n}(x, x)$. An equivalent statement has been given in [D-R1, Th. 3].

In the rest of this section we will construct an explicit bijection $\Pi$ : $\mathcal{P}_{2 n} \longrightarrow \mathcal{G}_{2 n}$ such that $(\operatorname{maxi} p, \operatorname{maxp} p)=(\operatorname{comi} \Pi(p), \operatorname{cemi} \Pi(p)), \quad p \in$ $\mathcal{P}_{2 n}$. Our bijection is similar to the one used by Dumont [D], see also Viennot $[\mathrm{V}]$.

A mapping $f:\{1,2, \ldots, n\} \longrightarrow\{0,1, \ldots, n-1\}$ is said to be subexcedent on $\{1,2, \ldots, n\}$, if $0 \leq f(i)<i$ for $i \in\{1,2, \ldots, n\}$. Let $\mathcal{F}_{n}$ be the set of sub-excedent mappings on $\{1,2, \ldots, n\}$ and $\mathcal{F}_{n}^{\prime}$ the set of subexcedent mappings on $\{1,2, \ldots, n\}$ with $f(\{1, \ldots, n\})=\{2 i: 0 \leq i \leq n\}$.

Proposition 3. There is a bijection $T: \mathcal{P}_{2 n} \longrightarrow \mathcal{F}_{2 n+1}^{\prime}$ such that for $p \in \mathcal{P}_{2 n}, T(p)(k)=0$ iff $p(2 n+1-k)=2 n$ for all $k \in\{2,3, \ldots, 2 n\}$.

Proof: For each $p \in \mathcal{P}_{2 n}$, we define the sub-excedent mapping $f \in \mathcal{F}_{2 n+1}^{\prime}$ by $f(i)=2 n-p(2 n-i+1)$ for $i \in\{1,2, \ldots, 2 n\}$ and $f(2 n+1)=2 n$. It is clear that this is a desired bijection.

Recall that for a $\sigma \in \mathcal{S}_{n}$, the value $x=\sigma(i)$ is said to be a fall (resp. rise) of $\sigma$ if $\sigma(i)>\sigma(i+1)$ (resp. $\sigma(i)<\sigma(i+1))$.

Proposition 4. There is a bijection $\eta: \mathcal{S}_{n} \longrightarrow \mathcal{F}_{n}$ such that if $\sigma \in S_{n}$ and $f=\eta(\sigma)$, then for all $x \in\{1,2, \ldots, n\}$, (i) $x$ is a left-to-right minimum iff $f(x)=0$; (ii) $x$ is a fall or $x=\sigma(n)$ iff $x \notin f(\{1,2, \ldots, n\})$.

Proof: For $\sigma \in \mathcal{S}_{n}$, we define $f=\eta(\sigma)$ as follows. For each $x \in$ $\{1,2, \ldots, n\}$, let $x=\sigma(i)$ with $i \in\{1,2, \ldots, n\}$, then

$$
f(x)= \begin{cases}0, & \text { if } x \text { is a left-to-right minimum, } \\ \sigma(k), & \text { otherwise and } k=\max \{j \mid j<i, \sigma(j)<\sigma(i)\} .\end{cases}
$$

The inverse mapping can be constructed by using the notion of forest of increasing trees representing the sub-excedent mappings of $\mathcal{F}_{n}$. We refer the reader to $[\mathrm{V}]$ for further details of this bijection.

Let $\mathcal{G}_{2 n}^{\prime}$ be the set of permutations in $\mathcal{S}_{2 n}$ whose rise set is all odd integers $\leq 2 n$, i.e $\{1,3, \ldots, 2 n-1\}$.

Proposition 5. There is a bijection $V: \mathcal{F}_{2 n+1}^{\prime} \longrightarrow \mathcal{G}_{2 n}^{\prime}$ such that if $f \in \mathcal{F}_{2 n+1}^{\prime}$ and $V(f)=\sigma$, then $k(1 \leq k \leq 2 n)$ is a left-to-right minimum of $\sigma$ iff $f(k+1)=0$.

Proof: By Proposition 3, the inverse mapping $\eta^{-1}$ maps $\mathcal{F}_{2 n+1}^{\prime}$ to the set of permutations $\sigma$ in $\mathrm{S}_{2 n+1}$ with rise set $\{2,4, \ldots, 2 n\}$. But for such a 
permutation $\sigma$ we must have $\sigma(2 n+1)=1$, otherwise 1 would be a rise. Now applying the transformation $\epsilon: \sigma(i) \mapsto \sigma(i)-1(1 \leq i \leq 2 n\})$ to each preceding permutation $\sigma$ leads to a permutation in $\mathcal{G}_{2 n}^{\prime}$. Therefore $V=\epsilon \circ \eta^{-1}$ is a desired bijection.

Proposition 6. There is a bijection $F: \mathcal{G}_{2 n}^{\prime} \longrightarrow \mathcal{G}_{2 n}$ such that $k(1 \leq$ $k \leq 2 n)$ is a left-to-right minimum of $\sigma$ iff $k$ is a cycle minimum of $F(\sigma)$

Proof: This is a variant of the well-known transformation of Foata (see $[\mathrm{F}-\mathrm{S}])$. We describe briefly the inverse mapping $\phi: \mathcal{G}_{2 n} \longrightarrow \mathcal{G}_{2 n}^{\prime}$ as follows. For each $\sigma \in \mathcal{G}_{2 n}$ we put the smallest number in each cycle at the begining of the cycle, and write the cycles in decreasing order of their first entries. This defines the canonical cycle decomposition of $\sigma$. If we remove the parentheses from the canonical cycle decomposition of $\sigma$, we obtain the permutation $\phi(\sigma) \in \mathcal{G}_{2 n}^{\prime}$. It is clear that $x=\sigma(i)(1 \leq i \leq 2 n)$ is a left-to-right minimum of $\phi(\sigma)$, iff $x$ is a cycle-minimum of $\sigma$.

For instance, if $\sigma=42716583 \in \mathcal{G}_{8}$, then the canonical factorisation is $\sigma=(5,6)(3,7,8)(2)(1,4)$ and $\phi(\sigma)=56378214$. The latter is a permutation has 3 (resp. 1) odd (resp. even) left-to-right minima whose rise set is $\{1,3,5,7\}$.

Proposition 7. There is a bijection $\Pi: \mathcal{P}_{2 n} \longrightarrow \mathcal{G}_{2 n}$ such that

$$
(\operatorname{maxi} p, \operatorname{maxp} p)=(\operatorname{comi} \Pi(p), \operatorname{cemi} \Pi(p)), \quad p \in \mathcal{P}_{2 n} .
$$

Proof: By combining Propositions 3, 5 and 6 , we see that $\Pi=F \circ V \circ T$ is such a bijection.

We illustrate the bijection in the following schema :

$$
\mathcal{P}_{2 n} \stackrel{T}{\longrightarrow} \mathcal{F}_{2 n+1}^{\prime} \stackrel{\eta^{-1}}{\longrightarrow} \mathcal{S}_{2 n+1} \stackrel{\epsilon}{\longrightarrow} \mathcal{G}_{2 n}^{\prime} \stackrel{F}{\longrightarrow} \mathcal{G}_{2 n} .
$$

For example, if $n=2$ we have

$$
\begin{aligned}
& 2244 \longrightarrow 00224 \longrightarrow 24531 \longrightarrow 1342 \longrightarrow(1342)=3142, \\
& 2444 \longrightarrow 00024 \longrightarrow 32451 \longrightarrow 2134 \longrightarrow(2)(134)=3241, \\
& 4244 \longrightarrow 00204 \longrightarrow 45231 \longrightarrow 3412 \longrightarrow(12)(34)=2143 .
\end{aligned}
$$

Proposition 8. For $n \geq 1$ we have

$$
D_{n}(x, y)=\sum_{\sigma \in \mathcal{G}_{2 n}} x^{\operatorname{comi} \sigma} y^{\operatorname{cemi} \sigma} .
$$

Proof: This follows from Proposition 2 and 7 .

Remark: We can also derive the identity

$$
\sum_{p \in \mathcal{P}_{2 n}} x^{\operatorname{maxi} p} y^{\operatorname{maxp} p}=\sum_{\sigma \in \mathcal{G}_{2 n}} x^{\operatorname{comi} \sigma} y^{\operatorname{cemi} \sigma}
$$


as follows. For $p \in \mathcal{P}_{2 n}$, the value $2 i(1 \leq i \leq n)$ is said to be a doubled fixed point of $p$ if there is a $j \neq 2 i(1 \leq j \leq n)$ such that $p(j)=p(2 i)=2 i$. Let $\mathrm{fd} p$ (resp. fnd $p$ ) be the number of doubled (resp. no doubled) fixed points of $p$. In [R1, Cor. 9, R-Z, Prop. 14] it was proved by induction that

$$
\sum_{p \in \mathcal{P}_{2 n}} x^{\operatorname{maxi} p} y^{\operatorname{maxp} p}=\sum_{p \in \mathcal{P}_{2 n}} x^{\mathrm{fd} p} y^{\mathrm{fnd} p} .
$$

On the other hand, it was proved bijectively in [D-R2, Prop. 1.3] that

$$
\sum_{p \in \mathcal{P}_{2 n}} x^{\mathrm{fd} p} y^{\mathrm{fnd} p}=\sum_{p \in \mathcal{G}_{2 n}} x^{\mathrm{cmax} p} y^{\mathrm{fix} p}
$$

Formula (2.4) follows then by noting that for $\sigma \in \mathcal{G}_{2 n}$, we have $\operatorname{cmax} \sigma=$ $\operatorname{comi} \sigma$ and $\operatorname{cemi} \sigma=$ fix $\sigma$.

\section{A continued fraction expansion}

A Dyck path of length $2 n(n \geq 1)$ is a sequence of nonnegative integers $\gamma=\left(\gamma_{0}, \ldots, \gamma_{2 n}\right)$ such that $\gamma_{0}=\gamma_{2 n}=0, \gamma_{i}-\gamma_{i-1}=+1$ ou -1 for all $i \in\{1,2, \ldots, 2 n\}$. We denote by $\Gamma_{2 n}$ the set of Dyck paths of length $2 n$. Let $\left(a_{n}\right)$ and $\left(b_{n}\right)(n \geq 0)$ be two given sequences. If each pair $\left(\gamma_{i-1}, \gamma_{i}\right)$ of $\gamma \in \Gamma_{2 n}(1 \leq i \leq n)$ is given the valuation

$$
v\left(\gamma_{i-1}, \gamma_{i}\right)= \begin{cases}a_{\gamma_{i-1}}, & \text { if } \gamma_{i}-\gamma_{i-1}=+1 \\ b_{\gamma_{i-1}}, & \text { if } \gamma_{i}-\gamma_{i-1}=-1\end{cases}
$$

then it is well-known (see $[F])$ that

$$
1+\sum_{n \geq 1} \sum_{\gamma \in \Gamma_{2 n}} \prod_{i=1}^{2 n} v\left(\gamma_{i-1}, \gamma_{i}\right) t^{n}=\frac{1}{1-\frac{a_{0} b_{1} t}{1-\frac{a_{1} b_{2} t}{1-\frac{a_{2} b_{3} t}{1-\frac{a_{3} b_{4} t}{\ddots}}}}}
$$

A Genocchi history of length $2 n$ is a pair $(\gamma, p)$ where $\gamma$ is a Dyck path of length $2 n$ and $p=\left(p_{1}, \ldots, p_{2 n}\right)$ is a sequence of $2 n$ integers such that $0 \leq p_{i} \leq\left\lceil\gamma_{i-1} / 2\right\rceil-1$ (resp. $\left.0 \leq p_{i} \leq\left\lceil\gamma_{i-1} / 2\right\rceil\right)$ if $\gamma_{i}-\gamma_{i-1}=-1$ (resp. $\gamma_{i}-\gamma_{i-1}=+1$ ). We denote by $\mathcal{H}_{2 n}$ the set of Genocchi histories of length $2 n$. 
THE ELECTRonic JoURnal of Combinatorics 3 (2) (1996), \#R22

Proposition 9. There is a bijection $\Psi: \mathcal{G}_{2 n} \longrightarrow \mathcal{H}_{2 n}$ such that if $\Psi(\sigma)=$ $(\gamma, p)$, then

$$
\gamma_{i}-\gamma_{i-1}= \begin{cases}-1, & \text { if } i \in\{\sigma(1), \sigma(3), \ldots, \sigma(2 n-1)\} \\ 1, & \text { if } i \in\{\sigma(2), \sigma(4), \ldots, \sigma(2 n)\}\end{cases}
$$

and

(ii) $\quad p_{i}= \begin{cases}\mid\left\{j: 1 \leq j<\sigma^{-1}(i) \text { and } \sigma(j)>i\right\} \mid, & \text { if } \gamma_{i}-\gamma_{i-1}=-1 \\ \mid\left\{j: \sigma^{-1}(i)<j \leq 2 n \text { and } \sigma(j)<i\right\} \mid, & \text { if } \gamma_{i}-\gamma_{i-1}=+1 .\end{cases}$

Proof: The proposition itself gives the construction of this bijection. In fact, it is easy to see that (i) defines a Dyck path. Let $i_{1}<i_{2}<\ldots<i_{n}$ and $j_{1}<j_{2}<\ldots<j_{n}$ be the ordered integers of $\{\sigma(2), \sigma(4), \ldots, \sigma(2 n)\}$ and $\{\sigma(1), \sigma(3), \ldots, \sigma(2 n-1)\}$ respectively. For each $\sigma \in \mathcal{G}_{2 n}(n \geq 1)$, we have $\sigma(2 k-1)>\sigma(2 k)(1 \leq k \leq n)$, so $i_{k}<j_{k}(1 \leq k \leq n)$. Therefore $\gamma_{i} \geq 0$ for $i=1, \ldots, 2 n$ and $\gamma_{2 n}=0$. We refer the reader to [R, Th. 6.2] for further details of this bijection.

Let

$$
R_{n}(x, y, \bar{x}, \bar{y})=\sum_{\sigma \in \mathcal{G}_{2 n}} x^{\mathrm{lema} \sigma} y^{\mathrm{romi} \sigma} \bar{x}^{\mathrm{fix} \sigma} \bar{y}^{\mathrm{remi} \sigma} .
$$

Proposition 10. We have the following continued fraction expansion :

$$
1+\sum_{n \geq 1} R_{n}(x, y, \bar{x}, \bar{y}) t^{n}=\frac{1}{1-\frac{x y t}{1-\frac{1(\bar{x}+\bar{y}) t}{1-\frac{(x+1)(y+1) t}{1-\frac{2(\bar{x}+\bar{y}+1) t}{1-\frac{(x+2)(y+2) t}{3(\bar{x}+\bar{y}+2) t}}}}}} .
$$

Proof: For each $\gamma \in \Gamma_{2 n}$ we denote by $\mathcal{G}_{2 n}(\gamma)$ the set of Genocchi permutations of $\mathcal{G}_{2 n}$ associated with $\gamma$ by the mapping $\Psi$. It is easy to see that the bijection $\Psi$ has the following properties :

(a) $2 i$ is a fixed point of $\sigma$ iff $\gamma_{2 i}-\gamma_{2 i-1}=+1$ and $p_{2 i}=\gamma_{2 i} / 2$;

(b) $\sigma(i)$ is a right-to-left minimum of $\sigma$ iff $\gamma_{2 i}-\gamma_{2 i-1}=+1$ and $p_{\sigma(i)}=0$;

(c) $\sigma(i)$ is a left-to-right maximum of $\sigma$ iff $\gamma_{2 i}-\gamma_{2 i-1}=-1$ and $p_{\sigma(i)}=0$; so that

$$
\left\{\begin{aligned}
\operatorname{fix} \sigma & =\left|\left\{2 i: \gamma_{2 i}-\gamma_{2 i-1}=+1, p_{2 i}=\gamma_{2 i} / 2\right\}\right| ; \\
\operatorname{remi} \sigma & =\left|\left\{2 i: \gamma_{2 i}-\gamma_{2 i-1}=+1, p_{2 i}=0\right\}\right| ; \\
\operatorname{romi} \sigma & =\left|\left\{2 i-1: \gamma_{2 i-1}-\gamma_{2 i-2}=+1, p_{2 i-1}=0\right\}\right| \\
\text { lema } \sigma & =\left|\left\{2 i: \gamma_{2 i}-\gamma_{2 i-1}=-1, p_{2 i}=0\right\}\right| .
\end{aligned}\right.
$$


Therefore

$$
\sum_{\sigma \in \mathcal{G}_{2 n}(\gamma)} x^{\text {lema } \sigma} y^{\text {romi } \sigma} \bar{x}^{\mathrm{fix} \sigma} \bar{y}^{\mathrm{remi} \sigma}=\prod_{i=1}^{2 n} v\left(\gamma_{i-1}, \gamma_{i}\right)
$$

where $v\left(\gamma_{i-1}, \gamma_{i}\right)(1 \leq i \leq n)$ is defined as follows :

$$
v\left(\gamma_{2 i-2}, \gamma_{2 i-1}\right)= \begin{cases}y+\frac{1}{2} \gamma_{2 i-2}, & \text { if } \gamma_{2 i-1}-\gamma_{2 i-2}=+1 \\ \frac{1}{2} \gamma_{2 i-2}+1, & \text { if } \gamma_{2 i-1}-\gamma_{2 i-2}=-1\end{cases}
$$

and

$$
v\left(\gamma_{2 i-1}, \gamma_{2 i}\right)= \begin{cases}\bar{x}+\bar{y}+\frac{1}{2}\left(\gamma_{2 i-1}-1\right), & \text { if } \gamma_{2 i}-\gamma_{2 i-1}=+1 \\ x+\frac{1}{2}\left(\gamma_{2 i-1}-1\right), & \text { if } \gamma_{2 i}-\gamma_{2 i-1}=-1\end{cases}
$$

Hence

$$
\begin{aligned}
1+\sum_{n \geq 1} t^{n} \sum_{\gamma \in \Gamma_{2 n}} \sum_{\sigma \in \mathcal{G}_{2 n}(\Gamma)} x^{\text {lema } \sigma} y^{\text {romi } \sigma} \bar{x}^{\mathrm{fix} \sigma} \bar{y}^{\mathrm{remi} \sigma}= \\
1+\sum_{n \geq 1} t^{n} \sum_{\gamma \in \Gamma_{2 n}} \prod_{i=1}^{2 n} v\left(\gamma_{i-1}, \gamma_{i}\right) .
\end{aligned}
$$

Now, formula (3.4) follows from (3.2) with the following values for the $a_{n}^{\prime} s$ and $b_{n}^{\prime} s$ :

$$
\begin{aligned}
a_{2 k-1} & =\bar{x}+\bar{y}+k-1, & a_{2 k-2} & =y+k-1, \\
b_{2 k-1} & =(x+k-1), & b_{2 k} & =k+1, \quad(k \geq 1) .
\end{aligned}
$$

Corollary 11. The polynomials $R_{n}(x, y, \bar{x}, \bar{y})(n \geq 1)$ are symmetric in $x, y$ and also in $\bar{x}, \bar{y}$.

Proof: It is obvious in view of the continued fraction expansion (3.4).

\section{Proof of Theorem 1 and a conjecture}

Proposition 12. A sequence of polynomials $P_{n}(x, y, \bar{x}, \bar{y})(n \geq 0)$ satisfies the recurrence:

$$
\left\{\begin{aligned}
P_{1}(x, y, \bar{x}, \bar{y}) & =x y \\
P_{n}(x, y, \bar{x}, \bar{y}) & =x(\bar{x}+\bar{y}) P_{n-1}(x+1, y, \bar{x}+1, \bar{y}) \\
& -x(\bar{x}+\bar{y}-y) P_{n-1}(x, y, \bar{x}, \bar{y})
\end{aligned}\right.
$$


if and only if

$$
\begin{aligned}
& 1+\sum_{n \geq 1} P_{n}(x, y, \bar{x}, \bar{y}) t^{n}= \\
& 1-\frac{1}{1-\frac{x y t}{1-\frac{1(\bar{x}+\bar{y}) t}{1-\frac{(x+1)(y+1) t}{1-\frac{(x+\bar{x}+1) t}{1-\frac{3(\bar{x}+\bar{y}+2) t}{\ddots}}}}} .}
\end{aligned}
$$

Proof : The reader is referred to [R-Z2, Th. 3].

It follows from Propositions 10 and 11 that $R_{n}(x, y, \bar{x}, \bar{y})(n \geq 1)$ satisfy recurrence (4.1). In particular $R_{n}(x, 1, y, 1)(n \geq 1)$ satisfies recurrence (1.4). In virtue of Corollary $9, R_{n}(1, x, 1, y)(n \geq 1)$ satisfy also recurrence (1.4). Combining with Proposition 8 of section 2 this completes the proof of Theorem 1.

Corollary 13. We have the following continued fraction expansion :

$$
1+\sum_{n \geq 1} \sum_{\sigma \in \mathcal{G}_{2 n}} x^{\mathrm{cyc} \sigma} t^{n}=\frac{1}{1-\frac{x t}{1-\frac{1(x+1) t}{1-\frac{2(x+1) t}{1-\frac{3(x+2) t}{1-\frac{3(x+2) t}{}}}}}} .
$$

Proof: It is straightforward by Theorem 1 and Proposition 10.

In view of Theorem 1, it would be interesting to consider the polynomials

$$
G_{n}(x, y, \bar{x}, \bar{y})=\sum_{\sigma \in \mathcal{G}_{2 n}} x^{\text {comi } \sigma} y^{\text {lema } \sigma} \bar{x}^{\text {cemi } \sigma} \bar{y}^{\text {remi } \sigma} \quad(n \geq 1) .
$$

Here are the first values of these polynomials :

$$
\begin{aligned}
G_{1}(x, y, \bar{x}, \bar{y})= & x y \\
G_{2}(x, y, \bar{x}, \bar{y})= & x y(x y+\bar{x}+\bar{y}) . \\
G_{3}(x, y, \bar{x}, \bar{y})= & x y\left[x^{2} y^{2}+(\bar{x}+\bar{y})(3 x y+x+y+1+\bar{x}+\bar{y})\right] . \\
G_{4}(x, y, \bar{x}, \bar{y})= & x y\left[x^{3} y^{3}+(\bar{x}+\bar{y})\left(6 x^{2} y^{2}+4 x^{2} y\right.\right. \\
& \left.+4 x y^{2}+8 x y+x^{2}+y^{2}+4 x+4 y+3\right) \\
& \left.+(\bar{x}+\bar{y})^{2}(7 x y+4 x+4 y+4)+(\bar{x}+\bar{y})^{3}\right] .
\end{aligned}
$$


We observe that $G_{n}(x, y, \bar{x}, \bar{y})$ is symmetric in $x, y$ and in $\bar{x}, \bar{y}$ for $n \leq 4$. We believe that this is true for all $n \geq 1$. More generaly, we have the following conjecture.

Conjecture 12. For $n \geq 1$ we have $G_{n}(x, y, \bar{x}, \bar{y})=R_{n}(x, y, \bar{x}, \bar{y})$.

Since $R_{n}(x, y, \bar{x}, \bar{y})(n \geq 1)$ is symmetric in $x, y$, the above conjecture can also be restated as follows.

Conjecture 12'. For $n \geq 1$ we have $G_{n}(x, y, \bar{x}, \bar{y})=R_{n}(y, x, \bar{x}, \bar{y})$, i.e,

$$
\sum_{\sigma \in \mathcal{G}_{2 n}}\left(x^{\text {comi } \sigma}-x^{\text {romi } \sigma}\right) y^{\text {lema } \sigma} \bar{x}^{\mathrm{fix} \sigma} \bar{y}^{\text {remi } \sigma}=0
$$

It is obvious that the conjecture is true if $x=1$ and Theorem 1 is equivalent to $G_{n}(x, 1, y, 1)=R_{n}(x, 1, y, 1)=R_{n}(1, x, 1, y)$ for all $n \geq 1$. Finally, this conjecture has been verified for $n \leq 4$.

\section{REFERENCES}

[A] André (D.): Développement de $\sec x$ et de $\tan x, C$. R. Acad. Sci. Paris, vol. 88, 1879, p. 965-967.

[C-S] Carlitz (L.) and Scoville (R.): Enumeration of up-down permutations by upper records, Monatsch. Math., vol. 79, 1975, p. 3-12.

[D] Dumont (D.): Interprétation combinatoire des nombres de Genocchi, Duke Math. J., vol. 41, 1974, p. 305-318.

[D-R1] Dumont (D.) and Randrianarivony (A.): Sur une extension des nombres de Genocchi , Europ. J. Combinatorics, vol. 16 , 1995, p. 147151 .

[D-R2] Dumont (D.) and Randrianarivony (A.): Dérangements et nombres de Genocchi, Discrete Math. , vol. 132, 1994, p. 37-49.

[D-V] Dumont (D.) and Viennot (G.) : A combinatorial interpretation of the Seidel generation of Genocchi numbers, Annals of Disc. Math., vol. 6, 1980, p. 77-87.

[F] Flajolet (P.): Combinatorial aspects of continued fractions , Disc. Math., vol. 32, 1980, p. 125-161.

[F-S] Foata (D.) and Schützenberger (M.): Théorie géométrique des polynômes Eulériens . Lecture Notes in Math., Vol. 138, SpringerVerlag, 1970.

[R1] Randrianarivony (A.): Polynômes de Dumont-Foata généralisés. Preprint, 1994 .

[R2] Randrianarivony (A.): Fractions continues, q-nombres de Catalan et q-polynômes de Genocchi. Preprint, 1995. 
[R-Z1] Randrianarivony (A.) and Zeng (J.): Sur une extension des nombres d'Euler et les records des permutations alternantes, J. Combin. Theory, Ser. A, vol. 68, 1994, p. 86-99.

[R-Z2] Randrianarivony (A.) and Zeng (J.): Une famille de polynômes qui interpole plusieurs suites classiques de nombres. To appear in Adv. Appl. Math. 1996.

[V] Viennot (G.): Théorie combinatoire des nombres d'Euler et Genocchi. Séminaire de Théorie des nombres, Exposé no. 11, Publications de l'Université de Bordeaux I. 1980’81. 